\title{
BIFURCATION TO QUASI-PERIODIC TORI IN THE INTERACTION OF STEADY STATE AND HOPF BIFURCATIONS*
}

\author{
JURGEN SCHEURLE ${ }^{\dagger}$ AND JERROLD MARSDEN ${ }^{\ddagger}$
}

\begin{abstract}
Bifurcations to quasi-periodic tori in a two parameter family of vector fields are studied. At criticality, the vector field has an equilibrium point with a zero eigenvalue and a pair of complex conjugate eigenvalues. This situation has been studied by Langford, Iooss, Holmes and Guckenheimer. Here we provide explicitly computed conditions under which the stability of the secondary branch of tori, and whether the flow on them is quasiperiodic, can be determined. The results are applied to "Brusselator" system of reaction diffusion equations.
\end{abstract}

Introduction. Consider a smooth vector field on $\mathbb{R}^{3}$ which has a singular point at the origin. Suppose that the linearized vector field at the origin has an eigenvalue zero and a pair of pure imaginary eigenvalues $\pm i \gamma, \gamma>0$. The aim of this paper is to show that in a two parameter unfolding of this singularity satisfying explicitly computed nondegeneracy conditions, there are continuous curves, emanating as a tertiary bifurcation from a secondary curve of Hopf periodic orbits, along which one has invariant 2-tori carrying quasi-periodic flow. In particular, this shows that within a structurally stable situation one has an abundance of bifurcations that are the first part of a Landau sequence:

$$
\begin{aligned}
& \text { trivial } \\
& \text { equilibrium }
\end{aligned} \rightarrow \begin{aligned}
& \text { primary } \\
& \text { branch of } \\
& \text { equilibria }
\end{aligned} \rightarrow \begin{aligned}
& \text { secondary branch } \\
& \text { of Hopf orbits }
\end{aligned} \rightarrow \begin{aligned}
& \text { tertiary branch of } \\
& \text { quasi-periodic 2-tori. }
\end{aligned}
$$

Explicit exchange of stability results are established for each bifurcation. Because of the concrete nature of the formulas for the nondegeneracy conditions that are derived, the results can be applied to specific problems for specific choices of parameters. We work out these conditions for a reaction diffusion problem as an example. We expect that the method will also apply to certain plasma instability problems; see Crawford [1983].

We shall work within the class of $C^{k}$ vector fields with a zero at the origin. No other symmetry conditions are imposed. The eigenvalues are assumed to cross the imaginary axis "with nonzero speed" with respect to the unfolding parameters and some nondegeneracy conditions are imposed on the second and third order terms of the unfolding. These assumptions will imply that the trivial solution undergoes transcritical and Hopf bifurcations. Langford [1979] showed that the interaction between this steady state and Hopf bifurcation leads to invariant tori under some generic-type assumptions. Although Langford's paper forms the basis for the present work, our approach is more in the spirit of singularity theory and the work of Holmes [1980] and Guckenheimer [1981], [1982] in that it uses normal forms and the ideas of unfolding. Of course there are now many papers in bifurcation theory using this approach, such as Golubitsky and Schaeffer [1979], Schaeffer and Golubitsky [1981] and Golubitsky and Langford [1981]. Some partial results similar in spirit to ours have been given by Broer [1982] (see also

*Received by the editors December 13, 1982, and in revised form September 26, 1983.

${ }^{\dagger}$ Universität Stuttgart, Pfaffenwaldring 57, D-7000 Stuttgart 80, West Germany. The research of the author was supported by Deutsche Forschungsgemeinschaft contract 13 Sche 233/2-1 while he was visiting the University of California, Berkeley.

‡Department of Mathematics, University of California, Berkeley, California 94720. The research of this author was partially supported by the U. S. Department of Energy under contract DE-AT03-82ER 12097. 
Broer [1981b], Braaksma, and Broer [1981], and Chow and Hale [1982]). As we have mentioned, we impose no symmetry conditions, but also do not forbid them. In particular, we begin with a normal form somewhat more general than that considered by Guckenheimer [1981], [1982].

To construct the bifurcating 2-tori, we use a theorem of Sacker [1965]. To locate the tori carrying quasi-periodic flow, we use KAM theory and methods of Scheurle [1982]. The results will be local, and will be robust against higher order perturbations, so we have a form of structural stability. (For global results, the work of Chenciner [1982] may be relevant.) Despite the fact that individual quasi-periodic flows are structurally unstable, their occurrence in this bifurcation is stable and in fact their occurrence along appropriate arcs in parameter space is an open condition. To make the explicit computation for the example considered in \$3, we use Poincaré-Birkhoff normal forms and center manifold theory, a technique of Ruelle and Takens [1971] that proved effective for explicit calculations in the Hopf bifurcation (see Marsden and McCracken [1976] Hassard and Wan [1978], and Hassard, Kazarinoff and Wan [1981]).

There are other singularities and corresponding unfoldings where our method should be applicable to yield invariant tori with quasi-periodic flow. If, for example, the spectrum of the linearized vector field is as above and the second order terms vanish identically, then we have a more degenerate singularity, and a two-parameter unfolding is reasonable only within the class of $\mathbb{Z}_{2}$-symmetric vector fields. In this context, one has an interaction between a pitchfork and a Hopf bifurcation. For this case, Langford and looss [1980] succeeded in showing the existence of invariant 2-tori provided the 5 -jet satisfies a certain (implicitly given) nondegeneracy condition. In the case of a vector field in $\mathbb{R}^{4}$ with two pairs of purely imaginary eigenvalues of the linearization, one expects the existence of invariant 3-tori under suitable conditions (see Iooss and Langford [1980], and Guckenheimer [1980]). However, as far as we know, only in some symmetric cases has it been shown that the flow on some of these tori is actually quasi-periodic. In particular, Guckenheimer [1980] assumes a type of axial symmetry and Broer [1981b] and Braaksma and Broer [1981] deal with divergence-free vector fields.

Although quasi-periodic motions are chaotic in some sense, one should also mention that even much more complicated dynamical behavior has been discovered in these problems. In the case considered in the present paper, Guckenheimer [1981], [1982] showed that a generic perturbation of a certain truncation of the system possesses transversal homoclinic orbits and hence horseshoes. He uses a geometric argument based on an argument of Silnikov. The precise hypotheses can be expected to be difficult to check in specific examples of perturbations. P. Holmes [1980] applied Melnikov's method to prove the existence of transversal homoclinic orbits for a very particular unfolding of this singularity. Exact verifiable results are again difficult to obtain since the Melnikov function is exponentially small and is not seen at finite orders in perturbation theory (cf. Holmes and Marsden [1982]). These results together with our result, however, strongly suggests the verifiable coexistence of both quasi-periodic motions and horseshoes, even for equal parameter values. We plan to address our attention to this question in a forthcoming paper.

The structure of the paper is as follows: In $\$ 1$ we discuss the normal form of the unfolding and we prove that there is a curve in the parameter space along which one has Neimark-Sacker bifurcations. We construct the bifurcating invariant tori near this curve and show that they are asymptotically stable if they bifurcate to the right, and unstable, if they bifurcate to the left. In $\$ 2$ we show that there are continuous curves in parameter space emanating from the critical curve, along which one has invariant tori 
with quasi-periodic flow. Finally in $\$ 3$ we apply our theory to a model system of reaction diffusion equations, the so-called Brusselator. Although this is a system of partial differential equations, center manifold theory and the method of BirkhoffPoincaré normal forms is used to reduce it to the normal form discussed before.

1. Bifurcation of periodic solutions into invariant 2-tori. We consider the following unfolding of a three-dimensional codimension-two singularity (cf. Guckenheimer [1981], [1982]):

$$
\begin{aligned}
& \dot{r}=(\lambda-\sigma) r+a r z+d r^{3}+e r z^{2}+\cdots+O(l), \\
& \dot{z}=\lambda z+b z^{2}+c r^{2}+f r^{2} z+g z^{3}+\cdots+O(l), \\
& \dot{\theta}=\gamma+h_{1} z+h_{2} r^{2}+h_{3} z^{2}+\cdots+O(l) / r .
\end{aligned}
$$

Here $(r, z, \theta)$ are cylindrical coordinates in $\mathbb{R}^{3}$. It is assumed that the vector field on the right is sufficiently smooth and has been written in Birkhoff-Poincaré normal form up to terms of order $l$, i.e. the coefficients $a, b, c, \cdots$ are real numbers, whereas $O(l)$ stands for functions which are of order $l$ in $r$ and $z$ uniformly in $\theta . \gamma$ is a given positive constant, while $\lambda$ and $\sigma$ are (real) unfolding parameters. We shall refer to $\lambda$ as the bifurcation parameter and to $\sigma$ as the splitting parameter (cf. Langford [1979]). Note that we cannot further simplify the $\theta$-equation as done by Guckenheimer [1981], [1982] or Broer [1982], since we are mainly interested in quasi-periodic solutions. Although invariant tori are preserved by these simplifications, Poincare rotation numbers are in general changed.

If $a b c \neq 0$ in (1.1), then the equation is classified by Langford [1979] into six qualitatively distinct cases according to the values of $a, b$, and $c$. In each case, if $\sigma \neq 0$, one has transcritical steady state bifurcations as well as Hopf bifurcations as $\lambda$ varies. These are the primary and secondary bifurcations. In one case the possibility of a so-called tertiary bifurcation is allowed, i.e. the bifurcation of periodic solutions into invariant 2-tori (through the Neimark-Sacker bifurcation). This case arises when

$$
a b<0 \text { and } c(b-a)>0 \text {. }
$$

Under an additional nondegeneracy condition on $d, e, f$, and $g$ given explicitly below in (1.14), we shall show in this section that such a bifurcation indeed occurs.

As far as primary and secondary bifurcations are concerned, there are three curves in the $(\lambda, \sigma)$-plane, along which such bifurcations occur, near the origin. These curves are given by the asymptotic formulas

$$
\begin{aligned}
& \bigodot_{1}: \lambda=O\left(\sigma^{2}\right), \\
& \bigodot_{2}: \lambda=\frac{b}{b-a} \sigma+O\left(\sigma^{2}\right), \\
& \bigodot_{3}: \lambda=\sigma+O\left(\sigma^{2}\right),
\end{aligned}
$$

respectively. Along $\mathcal{C}_{1}$ we have a transcritical stationary bifurcation from the trivial solution. For $\sigma>0$ the trivial solution loses its stability to the supercritical stationary solution branching from it. Along $C_{2}$ we have a Hopf bifurcation from the stable stationary solutions that branched out along $C_{1}$. For $\sigma>0$ this Hopf bifurcation is supercritical, and so the periodic solutions acquire the stability for a small parameter range. Finally, along $\mathcal{C}_{3}$ these periodic solutions run back into the trivial solution again in a subcritical Hopf bifurcation. Near this bifurcation point the periodic solutions are 
unstable. There is an intermediate neutral stability curve for the periodic solutions asymptotically given by

$$
\bigodot_{4}: \lambda=\frac{2 b}{2 b-a} \sigma+O\left(\sigma^{2}\right)
$$

Here the Floquet exponents of the periodic solutions are purely imaginary. This gives rise to a tertiary bifurcation into invariant 2-tori (cf. Marsden and McCracken [1976] and Iooss [1979] for expositions). See Fig. 1. For $\sigma<0$ the Hopf bifurcation from the trivial solution occurs first as in Fig. 1(c).

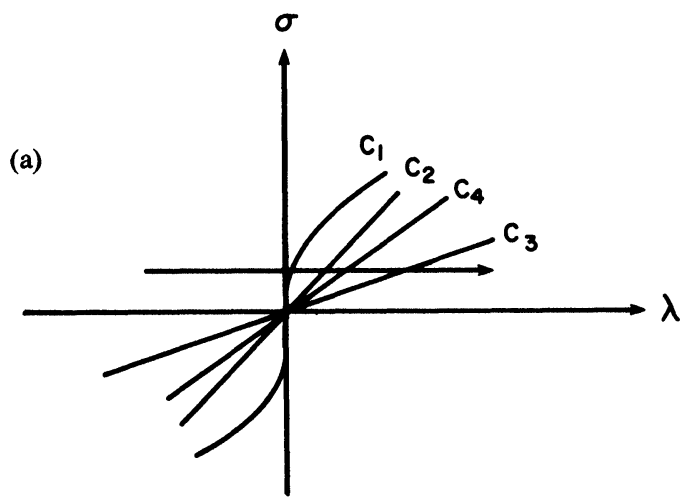

(b)

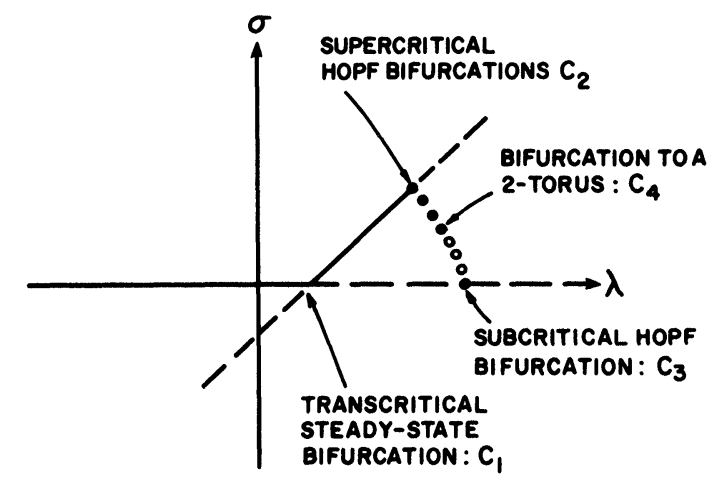

(c)

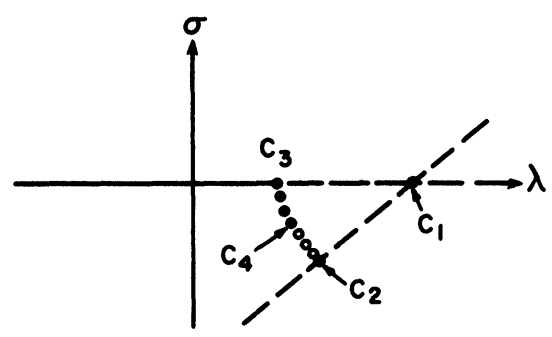

Fig. 1. (a) The curves $\mathcal{C}_{1}, \mathcal{C}_{2}, \mathcal{C}_{3}$ and $\mathcal{C}_{4}$ in the $(\lambda, \sigma)$-plane (b) The bifurcation diagram corresponding to the horizontal section shown in (a); $\sigma>0$. (c) The case $\sigma<0$. 
In order to construct these tori one proceeds as follows (see Guckenheimer [1982] for details): Truncate the equations in (1.1) after terms of order $l-1$. The truncated system is axisymmetric with respect to the $z$-axis and so the $\theta$-equation decouples from the $(r, z)$-part, and corresponding to the periodic solutions discussed above for $r \neq 0$ are the zeros (with $r \neq 0$ ) of the vector field $f=\left(f_{1}, f_{2}\right)$ given by

$$
\begin{aligned}
& f_{1}(\sigma, \lambda, r, z)=(\lambda-\sigma) r+a r z+\cdots+(\text { monomial of order } l-1), \\
& f_{2}(\sigma, \lambda, r, z)=\lambda z+b z^{2}+c r^{2}+\cdots+(\text { monomial of order } l-1) .
\end{aligned}
$$

Their "Floquet exponents" are given by the formula

$$
\mu_{ \pm}=\frac{1}{2} \operatorname{tr} D f \pm\left(\frac{1}{4} \operatorname{tr}^{2} D f-\operatorname{det} D f\right)^{1 / 2}
$$

Here $D f$ is the Jacobian matrix of $f$ with respect to $r$ and $z$. Hence, for the reduced system, the neutral stability curve for these solutions determined by the condition $\operatorname{Re} \mu_{ \pm}=0$, is given by

$$
f=0, \quad \operatorname{tr} D f=0,
$$

assuming det $D f>0$. In order to solve (1.6), we introduce rescaled variables as follows:

$$
\lambda=\sigma \tilde{\lambda}, \quad r=|\sigma| \tilde{r}, \quad z=\sigma \tilde{z}, \quad \tilde{r}>0,
$$

and consider the corresponding rescaled (or "blown-up") vector field

$$
\tilde{f}=\sigma^{-2} f \text {. }
$$

Note that there is a reflectional symmetry in our problem which guarantees that for given $\lambda$ and $\sigma$, solutions appear in pairs $(\theta, \pm r, z)$. Hence we can replace $|\sigma|$ by $\sigma$ in (1.7). For small $|\sigma|$, the implicit function theorem applies to yield a solution

$$
\tilde{\lambda}=\lambda_{0}(\sigma)=\frac{2 b}{2 b-a}+O(\sigma)
$$

of (1.6). Moreover, the implicit function theorem applies to yield the periodic orbits

$$
\tilde{r}=r_{0}(\sigma, \tilde{\lambda}), \quad \tilde{z}=z_{0}(\sigma, \tilde{\lambda})
$$

of $\tilde{f}$ for parameter values near the curve given by (1.9). Their Floquet exponents $\mu_{ \pm}=\alpha \pm i \beta$ have the properties

$$
\alpha\left(\sigma, \lambda_{0}(\sigma)\right) \equiv 0, \quad \frac{\partial \alpha}{\partial \tilde{\lambda}}\left(0, \lambda_{0}(0)\right)=\alpha_{0}=\frac{1}{2}-\frac{b}{a}>0, \quad \beta\left(0, \lambda_{0}(0)\right)=\beta_{0}>0 .
$$

All these functions are smooth (in fact locally analytic). Note that for practical reasons it suffices to compute them up to terms of order $l-1$ in $\sigma$.

Next we introduce local variables near the periodic orbits (1.10)

$$
\tilde{\lambda}=\lambda_{0}(\sigma)+\tilde{\Lambda}, \quad \tilde{r}=r_{0}(\sigma, \tilde{\lambda})+R, \quad \tilde{z}=z_{0}(\sigma, \tilde{\lambda})+Z,
$$

and transform the linearized vector field $D \tilde{f}$ to Jordan normal form. This can be achieved by a similarity transformation which is analytic in $\sigma$ and $\tilde{\Lambda}$. Furthermore, the transformed vector field is brought into Birkhoff-Poincaré normal form up to terms of order four. Again, this can be done via a coordinate transformation which is analytic in 
$\sigma$ and $\tilde{\Lambda}$. We end up with a vector field, the components of which have the following form in polar coordinates $(\tilde{\rho}, \phi), \tilde{\rho} \geq 0$, in the $(\tilde{R}, \tilde{Z})$ plane:

$$
\begin{aligned}
& \tilde{f}_{\tilde{\rho}}=\alpha \tilde{\rho}=A \tilde{\rho}^{3}+O\left(\tilde{\rho}^{5}\right), \\
& \tilde{f}_{\phi}=\beta+B \tilde{\rho}^{2}+O\left(\tilde{\rho}^{4}\right) .
\end{aligned}
$$

Here $\alpha$ and $\beta$ are as in (1.11), $A$ and $B$ are functions of $\sigma$ and $\tilde{\Lambda}$, and the symbols $O\left(\tilde{\rho}^{5}\right)$ and $O\left(\tilde{\rho}^{4}\right)$ stand for functions which are of order five and four, respectively, uniformly in $\phi, \sigma$ and $\tilde{\Lambda}$ and which are $2 \pi$-periodic in $\phi$. All functions are smooth (in fact analytic). We have the following asymptotic formula for $A$ :

$$
A(\sigma, \tilde{\Lambda})=\frac{b}{(2 b-a)^{2}}\left[4 b\left(\frac{b}{a}-1\right) d-\frac{4 b c}{a} e-2 b f+6 c g\right] \sigma+O\left(|\tilde{\Lambda}|+\sigma^{2}\right) .
$$

This formula is obtained by straightforward calculations as outlined above. We also have $A(0,0)=0$. This is a consequence of the fact that the vector field in $(1.8)$ is Hamiltonian and integrable for $\sigma=0$ and $\tilde{\lambda}=\lambda_{0}(0)$ (see Fig. 2, Guckenheimer [1982] and Langford [1982]). We shall make the following hypothesis:

$$
\frac{\partial}{\partial \sigma} A(0,0)=\Omega \neq 0 \text {. }
$$

For given values of $a, b, c$, and $h_{1}$, this is a nondegeneracy assumption for the third order terms in (1.1). Formula (1.14) tells us that this assumption is fulfilled for all values of the coefficients $h_{2}$ and $h_{3}$, and for $(d, e, f, g)$ outside a hyperplane in $\mathbb{R}^{4}$. Thus it is generic within the class of vector fields considered here.

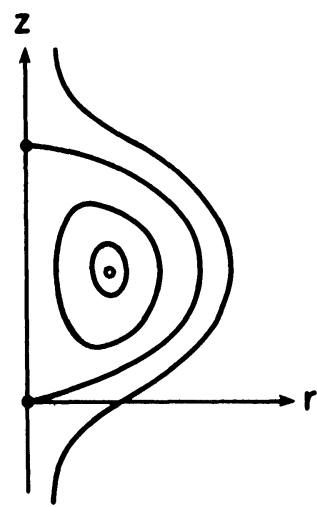

FIG. 2. The homoclinic orbit and phase portrait for $\tilde{f}$ with $\sigma=0$ and $\tilde{\lambda}=\lambda_{0}(0)$.

If we neglect the higher order terms in (1.13) for a moment, zeros of the equation

$$
\alpha \tilde{\rho}+A \tilde{\rho}^{3}=0
$$

correspond to limit cycles of that system. Moreover, if we add the equation $\dot{\theta}=\gamma$, they correspond to invariant 2-tori of the $(\theta, \tilde{r}, \tilde{z})$-system. In fact, we shall use these tori as a zeroth approximation for the invariant tori of the complete system.

Because of (1.11) and (H2) we can write

$$
\alpha(\sigma, \tilde{\Lambda})=\tilde{\Lambda} \tilde{\alpha}(\tilde{\sigma}, \tilde{\Lambda}), \quad A(\sigma, \tilde{\Lambda})=\sigma A_{1}(\sigma, \tilde{\Lambda})+\tilde{\Lambda} A_{2}(\sigma, \tilde{\Lambda}),
$$


where the functions $\tilde{\alpha}, A_{1}$ and $A_{2}$ are smooth (even analytic), and

$$
\tilde{\alpha}(0,0)=\alpha_{0}>0, \quad A_{1}(0,0)=\Omega \neq 0 .
$$

Hence, if $|\tilde{\Lambda}|$ is sufficiently small depending on $\sigma$, then (1.15) has a solution

$$
\rho_{0}=\sqrt{\frac{-\tilde{\Lambda} \tilde{\alpha}}{\sigma A_{1}+\tilde{\Lambda} A_{2}}},
$$

either for $\tilde{\Lambda} \geq 0$ if $\sigma \Omega<0$, or for $\tilde{\Lambda} \leq 0$ if $\sigma \Omega>0$. Thus, a vertical bifurcation of tori is excluded by our assumptions. Observe that $\rho_{0} \rightarrow 0$ as $\tilde{\Lambda} \rightarrow 0$.

In order to continue these tori to the complete system, we now introduce the rescaled parameter $\Lambda$ via

$$
\tilde{\Lambda}=\sigma^{m} \Lambda, \quad \delta \leq|\Lambda| \leq 1, \quad \Lambda \Omega<0 .
$$

where $m \geq 1$ is an odd integer to be determined later and $\delta$ is some given positive number. The restriction of $\Lambda$ to such a domain is quite natural, because $\tilde{\Lambda}=0$ corresponds to the neutral stability curve of the truncated system and differs from $\bigodot_{4}$ to a higher order in $\sigma$. Moreover, we restrict the $\tilde{\rho}$-variable to a neighborhood of the tori given by (1.18), i.e. we introduce a local action variable $\rho$ via

$$
\tilde{\rho}=\rho_{0}+\sigma^{n}|\Lambda|^{1 / 2} \rho, \quad|\rho| \leq 1,
$$

where the integer $n \geq m$ will be chosen later.

After making the above substitutions of variables in (1.1) (the $\theta$-variable is left unchanged so far), we end up with a system of the following type:

$$
\begin{aligned}
& \dot{\rho}=\sigma\left\{2 \rho_{0}^{2} A \rho+g_{1}(\sigma, \Lambda, \phi, \rho)+h_{1}(\sigma, \Lambda, \theta, \phi, \rho)\right\} \\
& \dot{\phi}=\sigma\left\{\beta+B \rho_{0}^{2}+g_{2}(\sigma, \Lambda, \phi, \rho)+h_{2}(\sigma, \Lambda, \theta, \phi, \rho)\right\} \\
& \dot{\theta}=\gamma+\sigma\left\{f_{0}(\sigma, \Lambda, \phi)+g_{3}(\sigma, \Lambda, \phi, \rho)+h_{3}(\sigma, \Lambda, \theta, \phi, \rho)\right\}
\end{aligned}
$$

Here the functions $\rho_{0}, \beta, A, B, f_{0}$ and $g_{k}(k=1,2,3)$ are analytic in the variables $\sigma$, $|\Lambda|^{1 / 2}, \phi, \rho$ restricted to the indicated regions. Furthermore, $f_{0}$ and $g_{k}$ are $2 \pi$-periodic trigonometric polynomials of finite degree in $\phi . f_{0}$ can in fact be chosen so that it has degree two by absorbing the higher order terms in $g_{3}$. Moreover, these functions have the following order of magnitude with respect to $\sigma$, uniformly in the other variables as $|\sigma| \rightarrow 0$ :

$$
\begin{aligned}
& \rho=O\left(|\sigma|^{(m-1) / 2}\right), \quad \beta=O(1), \\
& A=O(|\sigma|), \quad B=O(1), \\
& f_{0}=O(1), \quad g_{k}=O\left(|\sigma|^{n}+|\sigma|^{5(m-1) / 2-n}\right) \quad(k=1,2,3) .
\end{aligned}
$$

The functions $h_{k}(k=1,2,3)$ are as smooth as the original vector field with respect to $\theta, \phi, \rho$ and are at least continuous in the parameters, provided that $n$ is not too large compared with $l$. They are $2 \pi$-periodic in both the variables $\phi$ and $\theta$, and

$$
h_{k}=O\left(|\sigma|^{l-2}+|\sigma|^{l-n-1}\right) \quad \text { as }|\sigma| \rightarrow 0 .
$$


Observe that without loss of generality we can assume that $f_{0}$ does not explicitly depend on $\phi$. For otherwise, we transform the $\theta$-variable via

$$
\theta=\tilde{\theta}+\Psi(\sigma, \Lambda, \phi),
$$

where $\Psi$ is the solution of the equation

$$
\frac{\partial \Psi}{\partial \phi}=\frac{f_{0}-\left[f_{0}\right]}{\beta+B \rho_{0}^{2}}
$$

Here $\left[f_{0}\right]$ denotes the constant term of the trigonometric polynomial $f_{0}$. Clearly (1.25) has a unique solution, and this is again a $2 \pi$-periodic trigonometric polynomial in $\phi$ with coefficients depending smoothly (even analytically) on $\sigma$ and $|\Lambda|^{1 / 2}$. The new $\theta$-equation reads as follows:

$$
\dot{\tilde{\theta}}=\gamma+\sigma\left[f_{0}\right]+\sigma\left\{g_{3}+h_{3}\right\}-\sigma \frac{\partial \Psi}{\partial \phi}\left\{g_{2}+h_{2}\right\} .
$$

The other equations in (1.21) are not affected by this transformation. Subsequently we shall simply assume that $f_{0}$ does not depend on $\phi$ in (1.21).

Now we are ready to apply Sacker's theorem (Sacker [1965, Thm. 1]) on invariant submanifolds to prove the following result:

THEOREM 1.1. Let the vector field in (1.1) be of class $C^{r}$ with $r \geq 21$. Moreover, let the coefficients $a, b, c, \cdots$ satisfy the hypotheses $(\mathrm{H} 1)$ and $(\mathrm{H} 2)$. Let $\delta \in(0,1)$ be some given number and denote by $S_{\delta}^{ \pm}$the regions of the $(\lambda, \sigma)$-plane which are bounded by the two curves $\lambda=\sigma \lambda_{0}(\sigma) \pm \delta \sigma^{10}$ and $\lambda=\sigma \lambda_{0}(\sigma) \pm \sigma^{10}$, where $\lambda_{0}(\sigma)$ is as in (1.9). Then, there is $a$ positive $\sigma_{0}$ such that, for all parameter values either in $S_{\delta}^{+} \cap\left\{(\lambda, \sigma) \| \sigma \mid \leq \sigma_{0}\right\}$ or in $S_{\delta}^{-} \cap$ $\left\{(\lambda, \sigma)|| \sigma \mid \leq \sigma_{0}\right\}$ depending on the sign of $\Omega$, the system (1.1) possesses an invariant 2-torus of class $C^{r-1}$. This torus depends continuously on the parameters. If $\Omega>0$ it exists in $S_{\delta}^{+}$ and is locally attractive. If $\Omega<0$ it exists in $S_{\delta}^{-}$and is locally repelling. (Thus we have the usual exchange of stability phenomenon.)

Proof. We apply Sacker's theorem to the transformed system (1.21). To this end we set $l=21, m=9, n=10$ for the integers in (1.1), (1.19) and (1.20). Then, in Sacker's notation we have $u=\rho, x_{1}=\phi, x_{2}=\theta, \mu=\sigma^{10}, \omega_{1}=\sigma \beta+\sigma B \rho_{0}^{2}, \omega_{2}=\gamma+\sigma f_{0}, P_{1}=$ $\sigma^{2} \rho_{0}^{2} A / \sigma^{10}, \hat{A}_{1}=\left\{g_{2}+h_{2}\right\} / \sigma^{9}, \hat{A}_{2}=\left\{g_{3}+h_{3}\right\} / \sigma^{9}$, and $\hat{G}=\left\{g_{1}+h_{1}\right\} / \sigma^{9}$. So we are in his degenerate case $\mathrm{i})$. Because of $(\mathrm{H} 2), P_{1}$ is strictly bounded away from zero. The sign of $P_{1}$ is equal to that of $\Omega$. Furthermore, (1.22) and (1.23) imply that the functions $\hat{A}_{k}$ and $\hat{G}$ together with all their derivatives with respect to $\theta, \phi, \rho$, up to order $r$ tend to zero uniformly as $\sigma \rightarrow 0$. Hence, for $\delta \leq|\Lambda| \leq 1$ and $|\Lambda|$ sufficiently small Sacker's theorem applies to yield an invariant manifold of (1.21) given by a function

$$
\rho=\tau(\sigma, \Lambda, \theta, \phi),
$$

which is $C^{r-1}$ and $2 \pi$-periodic in $\theta$ and $\phi$ and together with its derivatives continuous in $\Lambda$ and $\sigma$. Note that the estimates in Sacker's proof are independent of $\omega=\left(\omega_{1}, \omega_{2}\right)$ in bounded regions. Hence it does not matter that $\omega$ depends on $\sigma$ in our case. Now, in view of (1.19), $\Lambda$ has to be chosen positive if $\Omega<0$ and negative if $\Omega>0$. This proves the two alternatives in the existence part of the theorem. In order to prove the stability assertion, note that the constructed torus is locally stable if $-\mu P_{1}$ is positive i.e., if $\Omega<0$ (cf. (1.16)) and unstable if this matrix is negative, i.e. if $\Omega>0$.

Remark 1.2. The regularity assumption in Theorem 1.1 is obviously not optimal. Moreover, the existence domain of the tori in the $(\lambda, \sigma)$-plane is only estimated very 
roughly. It cannot be expected in general that the tori reach the curve $\lambda=\sigma \lambda_{0}(\sigma)$, i.e. $\delta=0$, for this curve is only an approximate neutral stability curve for the periodic solutions of (1.1). However, we still aim to show that the tori actually emanate along curves from the exact neutral stability curve $\bigodot_{4}$.

To prove this, one has to localize the vector field around the exact periodic solutions of (1.1) rather than around approximate solutions as in (1.12). Moreover, the complete 2-jet of the localized vector field has to be taken into consideration for the construction of a zeroth approximation of the tori. To this end, one has to transform away all nonresonant terms of the 2-jet. In fact, there is an almost identical transformations of the variables $\tilde{\Lambda}, R, Z, \theta$ of the form

$$
\begin{aligned}
& \tilde{\Lambda}=\tilde{\Lambda}+\Lambda_{0}(\sigma), \\
& R=\tilde{R}+R_{0}(\sigma, \tilde{\Lambda}, \tilde{\theta}, \tilde{R}, \tilde{Z}), \\
& Z=\tilde{Z}+Z_{0}(\sigma, \tilde{\Lambda}, \tilde{\theta}, \tilde{R}, \tilde{Z}), \\
& \theta=\tilde{\theta}+\psi_{0}(\sigma, \tilde{\Lambda}, \tilde{\theta}, \tilde{R}, \tilde{Z})
\end{aligned}
$$

such that, in the new variables, our method applies uniformly for $|\tilde{\tilde{\Lambda}}| \lesssim 1$ and $|\sigma|$ sufficiently small. The periodic solutions are given by $\tilde{R}=0, \tilde{Z}+=0$, and $\tilde{\tilde{\Lambda}}=0$ corresponds to the curve $\bigodot_{4}$. The remaining resonant terms in the 2-jet (with respect to $\tilde{R}$ and $\tilde{Z}$ ) of the transformed vector field are independent of $\tilde{\theta}$ and differ from the old ones only by terms of order greater than or equal to $l$ in $\sigma$. The same is true for higher order terms. In (1.28) all functions are smooth, $2 \pi$-periodic in $\tilde{\theta}$, and polynomial of order two in $\tilde{R}$ and $\tilde{Z}$. Such a transformation is easily constructed using the classical implicit function theorem, for there are no small divisors involved.

COROLlaRY 1.3. Let the assumptions of Theorem 1.1 hold. Then, for each fixed $\sigma$ sufficiently small, there is a continuous branch of invariant 2-tori of (1.1) which emanates at the curve $\mathcal{C}_{4}$ from periodic solutions. It can be parametrized by $\lambda$. For each parameter value, the torus contains the corresponding periodic orbit in its interior. (The torus collapses to the periodic orbit if the curve $\bigodot_{4}$ is approached.) It is locally stable if it bifurcates supercritically, and unstable if it bifurcates subcritically.

Remark 1.4. A result is similar to Corollary 1.3 has been proved by Langford [1979]. However, his condition is rather implicit, and it seems to be hard to check it in applications.

Remark 1.5. In many formulations for the bifurcation to tori, a nonresonance condition up to order four is needed. This does not occur here since the characteristic exponents of the periodic solutions tend to zero as $\sigma \rightarrow 0$ while their period tends to $\gamma>0$.

2. The existence of bifurcating tori with quasi-periodic flow. It is well known (cf. Iooss [1979]), that the flow on a bifurcating 2-torus is qualitatively described by Poincare's rotation number $p$. If $p$ is rational, then the flow is periodic and otherwise it is quasi-periodic, so ergodic. Also it is known that there is a set of parameter values of positive Lebesgue measure for which the flow is ergodic, provided that $p$ varies effectively with a parameter. The measure of this set tends to 1 when the corresponding family of flows approaches a family of parallel flows (see Arnol'd [1965] and Herman [1977]). The purpose of this section is to show that this actually happens in the present situation for generic paths through the $(\lambda, \sigma)$-plane close to the origin. In particular we shall see that there are specific continuous curves emanating from $\bigodot_{4}$ along which all the tori are quasi-periodic. In between these curves one expects the phenomenon of 
phase locking to occur which means that $p$ takes constant rational values in open regions of the parameter space (see Arnol'd [1965]).

Let $\rho=\tau(\sigma, \Lambda, \theta, \phi)$ be the invariant 2-manifold of (1.21) constructed in the previous section. Then the flow on this submanifold induced by (1.21) is given by the equations

$$
\dot{\phi}=\sigma\left\{\beta+B \rho_{0}^{2}+g_{2}+h_{2}\right\}, \quad \dot{\theta}=\gamma+\sigma\left\{f_{0}+g_{3}+h_{3}\right\}
$$

where the $\rho$-argument in the functions $g_{k}$ and $h_{k}$ (in (1.21)) is replaced by $\tau$. Rescaling the time by a factor $\kappa \sim 1$ we get

$$
\dot{\boldsymbol{\phi}}=\frac{\sigma}{\boldsymbol{\kappa}}\left\{\boldsymbol{\beta}+B \rho_{0}^{2}+g_{2}+h_{2}\right\}, \quad \dot{\boldsymbol{\theta}}=\frac{\gamma}{\kappa}+\frac{\sigma}{\kappa}\left\{f_{0}+g_{3}+h_{3}\right\} .
$$

Now, because $\beta\left(0, \lambda_{0}(0)\right)=\beta_{0}>0$ and $\gamma>0$, we can replace $\sigma$ and $\kappa$ in (2.2) by new parameters

$$
\omega_{1}=\frac{\sigma}{\kappa}\left(\beta+B \rho_{0}^{2}\right), \quad \omega_{2}=\frac{\gamma}{\kappa}+\frac{\sigma}{\kappa} f_{0} .
$$

Recall that $\rho_{0}^{2}$ is of order $O\left(||^{m-1}\right)$ as $\sigma \rightarrow 0$ and $f_{0}=O(1)$ can be made independent of $\phi$. Hence, when $m \geq 3$ the implicit function theorem gives a unique smooth solution

$$
\sigma=\sigma\left(\Lambda, \omega_{1}, \omega_{2}\right), \quad \kappa=\kappa\left(\Lambda, \omega_{1}, \omega_{2}\right)
$$

of equations (2.3) for $\omega_{1}$ near 0 and $\omega_{2}$ near $\gamma$. In particular, we have

$$
\sigma(\Lambda, 0, \gamma)=0, \quad \kappa(\Lambda, 0, \gamma)=1, \quad \sigma=O\left(\left|\omega_{1}\right|\right) \quad \text { as } \omega_{1} \rightarrow 0 .
$$

We shall look for curves in $\left(\omega_{1}, \omega_{2}\right)$-space parametrized by $\Lambda$, where the flow of (2.2) is quasi-periodic with two given basic frequencies $\left|\omega_{1}^{0}\right|$ and $\omega_{2}^{0}$ near 0 and $\gamma$, respectively ( $\omega_{1}^{0}$ is allowed to be negative). To this end we first consider the modified system

$$
\dot{\phi}=\omega_{1}^{0}+\Delta \omega_{1}+\frac{\sigma}{\kappa}\left\{g_{2}+h_{3}\right\}, \quad \dot{\theta}=\omega_{2}^{0}+\Delta \omega_{2}+\frac{\sigma}{\kappa}\left\{g_{3}+h_{3}\right\} .
$$

Note that the right-hand sides of these equations are smooth in $\phi$ and $\theta$ and, together with their derivatives, continuous in $\Lambda, \omega_{1}$ and $\omega_{2}$ (recall that we have only proved that $\tau$ is continuous in $\sigma$ and $\Lambda$ ).

LEMMA 2.1. Let the functions $g_{k}$ and $h_{k}$ be of class $C^{r}, r \geq 8$, in $\theta$ and $\phi$ and let them satisfy (1.22) and (1.23) with respect to $\sigma(l, m, n$ as in $\$ 1)$. Moreover, let $q$ be a given integer with $q \leq \min (n, 5(m-1) / 2-n, l-2, l-n-1)$. Then, there is a positive constant $c$ with the following property. If the vector $\omega^{0}=\left(\omega_{1}^{0}, \omega_{2}^{0}\right)$ is contained in the set

$$
I_{\varepsilon}=\left\{\omega^{0} \in \mathbb{R}|| j_{1} \omega_{1}^{0}+\left.j_{2} \omega_{2}^{0}|\geq \varepsilon| j\right|^{2} \text { for all } j \neq 0 \in \mathbb{Z}^{2}\right\}
$$

for some $\varepsilon \in(0,1)$, then there is a continuous function $\Delta \omega=\Delta \omega(\Lambda, \omega)$ defined for

$$
\left|\omega_{1}\right|<c \varepsilon^{2 /\{\min (n, 5(m-1) / 2-n, l-2, l-n-1)-q+1\}}
$$

and a $2 \pi$-periodic coordinate transformation of class $C^{r-3}$

$$
\boldsymbol{\phi}=\tilde{\boldsymbol{\phi}}+U(\Lambda, \omega, \tilde{\boldsymbol{\phi}}, \tilde{\boldsymbol{\theta}}), \quad \boldsymbol{\theta}=\tilde{\boldsymbol{\theta}}+V(\Lambda, \omega, \tilde{\boldsymbol{\phi}}, \tilde{\boldsymbol{\theta}})
$$

continuous in $\Lambda$ and $\omega$, such that (2.6) is transformed into

$$
\stackrel{\tilde{\phi}}{=}=\omega_{1}^{0}, \quad \tilde{\boldsymbol{\theta}}=\omega_{2}^{0} .
$$


In particular, we have

$$
\Delta \omega=O\left(\left|\omega_{1}\right|^{q}\right) \quad \text { as } \omega_{1} \rightarrow 0 .
$$

Proof. The existence of such a transformation is proved, for instance, by Zehnder [1975, Thm. 4.1] (cf. also Moser [1966] and Hörmander [1977]). The estimates (2.8) and (2.11) are due to the fact, that the functions $(\sigma / \kappa)\left\{g_{2}+h_{2}\right\} / \varepsilon^{2} \omega_{1}^{q}$ and $(\sigma / \kappa)\left\{g_{3}+\right.$ $\left.h_{3}\right\} / \varepsilon^{2} \omega_{1}^{q}$, together with their derivatives, have to be sufficiently small. Here the factor $\varepsilon^{2}$ in the denominator stems from the nonresonance condition (2.7) and the second factor $\omega_{1}^{q}$ guarantees (2.11). In view of (1.22), (1.23) and (2.5), these functions can be made arbitrarily small by choosing an appropriate $c$ in (2.8).

In particular, this lemma implies that the flow of the modified system (2.6) is quasi-periodic with the two basic frequencies $\omega_{1}^{0}$ and $\omega_{2}^{0}$ :

$$
\phi=\omega_{1}^{0} t+U\left(\Lambda, \omega, \omega_{1}^{0} t, \omega_{2}^{0} t\right), \quad \theta=\omega_{2}^{0} t+V\left(\Lambda, \omega, \omega_{1}^{0} t, \omega_{2}^{0} t\right) .
$$

Hence, it remains to be shown that there is a curve in $\left(\omega_{1}, \omega_{2}\right)$-space, on which the systems (2.2) and (2.6) agree. Such a curve is determined by solutions of the equations

$$
\omega_{1}=\omega_{1}^{0}+\Delta \omega_{1}\left(\Lambda, \omega_{1}, \omega_{2}\right), \quad \omega_{2}=\omega_{2}^{0}+\Delta \omega_{2}\left(\Lambda, \omega_{1}, \omega_{2}\right) .
$$

We shall solve (2.13) for $\omega_{1}$ and $\omega_{2}$ using Brouwer's fixed point theorem.

Let $\eta=\eta(\varepsilon)$ denote the right-hand side of the inequality in (2.8), and set $\omega_{0}=(0, \gamma)$. Then, with respect to $\omega$, the right-hand sides of the equations in (2.13) define a continuous map from the ball $B_{\eta}\left(\omega_{0}\right)$ with center $\omega_{0}$ and radius $\eta$, into $\mathbb{R}^{2}$. This map depends continuously on $\Lambda$. In order to make sure that it maps $B_{\eta}\left(\omega_{0}\right)$ into itself, we require

$$
\left|\omega^{0}-\omega_{0}\right| \leq \eta-\tilde{c} \eta^{q}
$$

where the constant $\tilde{c}$ stems from (2.11). Here a difficulty arises. Namely, it is not clear whether or not there are elements $\omega^{0}$ in $I_{\varepsilon}$ satisfying (2.14). But a measure-theoretical result helps. It is "well known" (see Siegel and Moser [1971] and Rüssmann [1979]) that the Lebesgue measure of the complement set of $I_{\varepsilon}$ in any ball is of order $O(|\varepsilon|)$ as $\varepsilon \rightarrow 0$, uniformly for balls with small radius, i.e. we have

$$
\mu\left(B_{\eta-\tilde{c} \eta^{q}}\left(\omega_{0}\right) \backslash I_{\varepsilon}\right) \leq \tilde{\tilde{c}} \varepsilon
$$

with some constant $\tilde{c}$, where $\mu$ denotes Lebesgue measure. Thus

$$
\pi\left(\eta-\tilde{c} \eta^{q}\right)^{2}-\tilde{c} \varepsilon>0
$$

implies

$$
B_{\eta-\tilde{c} \eta^{q}}\left(\omega_{0}\right) \cap I_{\varepsilon} \neq \varnothing
$$

Hence, if $q>1$ and $\eta$ is of order less than $\sqrt{\varepsilon}$ as $\varepsilon \rightarrow 0$, there are many frequency vectors $\omega^{0}$ such that Brouwer's fixed point theorem applies to yield a solution

$$
\omega=\omega(\Lambda)
$$

of (2.13) which depends continuously on $\Lambda$ (see Chow, Mallet-Paret and York [1978]). In fact, the relative measure of the set $B_{\eta-\tilde{c} \eta^{q}}\left(\omega_{0}\right) \cap I_{\varepsilon}$ in this ball tends to 1 as $\varepsilon \rightarrow 0$. 
THEOREM 2.2. Let the assumptions of Theorem 1.1 hold. Let $S_{\delta}^{ \pm}$be the regions of the $(\lambda, \sigma)$-plane as defined there, and let $\beta_{0}$ be as in (1.11). Then each interval around 0 contains a subset I with the following properties: The relative measure of I tends to 1 as the interval shrinks to the point 0 . For all $\sigma_{0} \in I$, near the line $\sigma=\sigma_{0}$ there is a continuous curve in $S_{\delta}^{ \pm}$

$$
\lambda=s_{ \pm}^{1}\left(\sigma_{0}, \Lambda\right), \quad \sigma=s_{ \pm}^{2}\left(\sigma_{0}, \Lambda\right) \quad(\delta \leq \Lambda \leq 1),
$$

along which the flow on the tori constructed in the previous section is quasi-periodic with two basic frequencies $\nu_{1} \sim \sigma_{0} \beta_{0}$ and $\nu_{2} \sim \gamma$. The frequency ratio $\nu_{1} / \nu_{2}$ is a constant along each curve. These curves join the two boundaries of $S_{\delta}^{ \pm}$and form a set of positive measure.

Proof. Let the integers $l, m, n$ be chosen as in the proof of Theorem 1.1 and set $q=2$. The right-hand side of the inequality in (2.8) is of order less than $\sqrt{\varepsilon}$ as $\varepsilon \rightarrow 0$. Thus, according to the above discussion and (2.4), for almost all frequency vectors $\omega^{0}$ near $\omega_{0}=(0, \gamma)$, there are values $\sigma=\sigma\left(\Lambda, \omega^{0}\right), \kappa=\kappa\left(\Lambda, \omega^{0}\right)$ depending continuously on $\Lambda$, such that the flow of (2.2) is quasi-periodic and given by (2.12). In view of (2.3) and (2.11), we have

$$
\left|\frac{\sigma \beta_{0}}{\kappa}-\omega_{1}^{0}\right|=O\left(|\sigma|^{2}\right), \quad\left|\frac{\gamma}{\kappa}-\omega_{2}^{0}\right|=O(|\sigma|) \quad \text { as } \sigma \rightarrow 0 .
$$

Now, consider the curves $\sigma=\sigma\left(\Lambda, \omega^{0}\right)$ in parameter space. In the original time scaling, the corresponding frequencies are $\nu_{1}=\kappa \omega_{1}^{0}$ and $\nu_{2}=\kappa \omega_{2}^{0}$. Hence, for different ratios $\omega_{1}^{0} / \omega_{2}^{0}$ these curves cannot intersect. However, if the ratios for two different $\omega^{0}$ are coincident, the corresponding curves may coincide. Therefore, it suffices to consider this subspace of $\omega^{0}$ 's where $\omega_{2}^{0}=\gamma$. In this case, (2.3) implies

$$
\kappa=1+O(|\sigma|) \text {. }
$$

Thus, with

$$
\sigma_{0}=\omega_{1}^{0} / \beta_{0}
$$

we conclude from $(2.20)$ that

$$
\left|\sigma-\sigma_{0}\right|-O\left(\left|\sigma_{0}\right|^{2}\right), \quad\left|\nu_{1}-\omega_{1}^{0}\right|=O\left(\left|\sigma_{0}\right|^{2}\right), \quad\left|\nu_{2}-\omega_{2}^{0}\right|=O\left(\left|\sigma_{0}\right|\right)
$$

as $\sigma_{0} \rightarrow 0$. Now set

$$
s_{ \pm}^{1}\left(\sigma_{0}, \cdot\right)=\sigma\left(\omega^{0}, \cdot\right), s_{ \pm}^{2}=s_{ \pm}^{1} \lambda_{0}\left(s_{ \pm}^{1}\right)+\left(s_{ \pm}^{1}\right)^{10} \Lambda
$$

with $\lambda_{0}$ from (1.9), and note that the measure theoretical result mentioned above, carries over to the subspace of frequency vectors $\omega^{0}$ with fixed second component $\omega_{2}^{0}=\gamma$. Thus, the theorem follows.

Remark 2.3. As in the case of Corollary 1.3 it can be shown that the curves in Theorem 2.2 actually emanate at the neutral stability curve $\mathcal{C}_{4}$ of the periodic solutions of (1.1). This has an interesting consequence. Passing through the parameter space on a path which runs into one of these curves, one has a bifurcation from periodic orbits into quasi-periodic solutions with two basic frequencies. See Fig. 3. These are stable if they are supercritical. Although individual quasi-periodic flows are nongeneric, one generically sees this bifurcation in the unfolding in (1.1) which thus exhibits the first part of the Landau sequence of transitions from trivial stationary solutions to quasiperiodic solutions. 


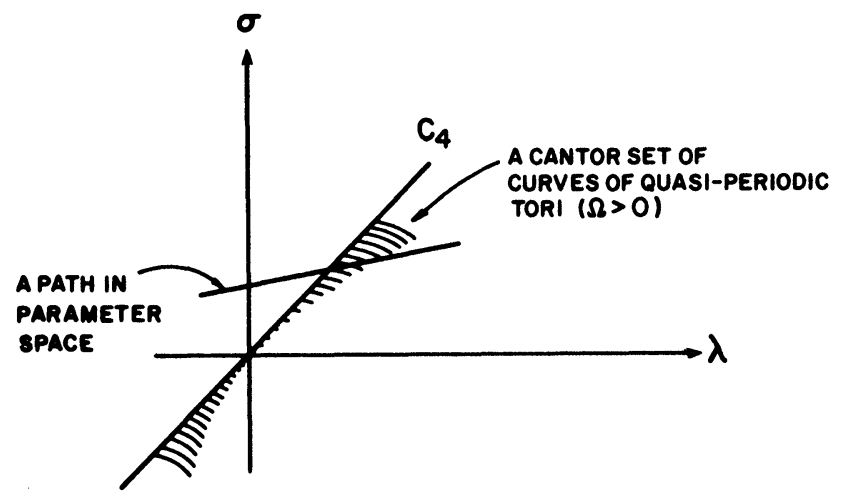

FIG. 3. A path in parameter space generally meets a set of positive measure of quasi-periodic tori.

Remark 2.4. Note, that for an analytic vector field in (1.1) the tori with quasi-periodic flow and frequency vectors contained in some set $I_{\varepsilon}$ are analytic 2-manifolds. This can, for instance, be proved by the method in Scheurle [1982], which combines the construction of the torus, with that of the flow on it and thus avoids a separate reduction step as in Theorem 1.1 (cf. also Bogoliubov, Mitropolskii and Samoilenko [1976]). In general, even $C^{\infty}$-smoothness is lost by such a reduction process (see Sacker [1965]).

Remark 2.5. Consider the differential equation

$$
x^{\prime}=f(\sigma, \lambda, x),
$$

where $f: \mathbb{R} \times \mathbb{R} \times \mathbb{R}^{3} \rightarrow \mathbb{R}^{3}$ is sufficiently smooth. Assume the existence of a trivial solution $x \equiv 0$, i.e. $f(\sigma, \lambda, 0) \equiv 0$. Moreover, let $D_{x} f(\sigma, \lambda, 0)$ have the eigenvalues $\alpha(\sigma, \lambda)$, and $\beta(\sigma, \lambda) \pm i \gamma(\sigma, \lambda)$ where $\alpha(0,0)=0, \beta(0,0)=0, \gamma(0,0) \neq 0$ and

$$
\frac{\partial(\alpha, \beta)}{\partial(\sigma, \lambda)} \neq 0 \quad \text { at }(\sigma, \lambda)=(0,0) \text {. }
$$

This is a generalized Hopf condition due to Langford [1979]. Then, introducing $\alpha$ and $\alpha-\beta$ as new parameters, (2.25) can be transformed to a system of form (1.1). Of course, the coefficients $a, b, c, \cdots$, and $\gamma$ will depend on the parameters in general. However, our theory still applies to this situation, if in (H1), (1.2), (1.3), (1.9), (1.11) and (1.14) the values of the coefficients at $\sigma=0, \lambda=0$ are inserted.

3. The Brusselator. We apply our theory to a model system of reaction diffusion equations, namely to the so-called Brusselator. Although this system originally consists of partial differential equations, there are parameter values where the solutions we are interested in, lie in a three-dimensional, locally invariant center manifold, and the restriction to this submanifold has a singularity of the type discussed in the previous sections. In particular, the generalized Hopf condition (2.26) is fulfilled, so that (1.1) is a reasonable unfolding. This example has been studied in detail by Guckenheimer [1982] (see also Keener [1976]). (See Schaeffer and Golubitsky [1981] for a discussion of the steady state bifurcations near a double zero eigenvalue.) We shall give explicit formulas for the relevant coefficients and in particular we compute the third order coefficients. We shall use a complex representation for convenience.

The following reaction schema is considered:

$$
A \rightarrow X, \quad B+X \rightarrow Y+D, \quad 2 X+Y \rightarrow 3 X, \quad X \rightarrow E .
$$


Here $A, B, D, E$ are reactants whose concentrations are assumed to be fixed throughout the reaction. It is the dynamics of the intermediates $X$ and $Y$ which is examined. In addition, the reaction is assumed to take place in a one-dimensional medium (with position variable $\xi$ ) and that $X$ and $Y$ diffuse with diffusion constants $D_{1}$ and $D_{2}$. This yields the following system of reaction diffusion equations

$$
\begin{aligned}
& \frac{\partial X}{\partial t}=D_{1} \frac{\partial^{2} X}{\partial \xi^{2}}+X^{2} Y-(B+1) X+A, \\
& \frac{\partial Y}{\partial t}=D_{2} \frac{\partial^{2} Y}{\partial \xi^{2}}-X^{2} Y+B X .
\end{aligned}
$$

It is further assumed that the reaction is at equilibrium at the end points of the interval $[0, \pi]$ so that $X(0)=X(\pi)=A$ and $Y(0)=Y(\pi)=B / A$ for all $t \geq 0$.

Obviously, the Brusselator problem has the trivial equilibrium solution $X(\xi, t)=A$, $Y(\xi, t)=B / A$. Let us introduce the relative coordinates

$$
u=X-A, \quad v=Y-B / A .
$$

Then the equations (3.1) become

$$
\begin{aligned}
& \frac{\partial u}{\partial t}=D_{1} \frac{\partial^{2} u}{\partial \xi^{2}}+(B-1) u+A^{2} v+\left(\frac{B}{A} u^{2}+2 A u v+u^{2} v\right) \\
& \frac{\partial v}{\partial t}=D_{2} \frac{\partial^{2} v}{\partial \xi^{2}}-B u-A^{2} v-\left(\frac{B}{A} u^{2}+2 A u v+u^{2} v\right)
\end{aligned}
$$

and the corresponding boundary conditions are $u(0, t)=u(\pi, t)=0$ and $v(0, t)=v(\pi, t)$ $=0$. If $w=(u, v)$, we write $w_{t}=L w+N(w)$, where $L w$ is the linear part of the right-hand side of (3.3). Let us consider $L$ as a linear operator in the Banach space $C^{0}[0, \pi]$ with domain of a definition equal to the subspace $C_{0}^{2}[0, \pi]$ of $C^{2}$-functions which are zero at the boundary of $[0, \pi]$. Representing $w$ as a Fourier series $w(\xi, t)=\sum_{n=1}^{\infty} w_{n}(t) \sin n \xi$, we find that the two-dimensional spaces spanned by the vector valued functions $w_{n} \sin n \xi$ are invariant for $L$ with spectrum given by the eigenvalues of

$$
E_{n}=\left(\begin{array}{cc}
B-1-n^{2} D_{1} & A^{2} \\
B & -A^{2}-n^{2} D_{2}
\end{array}\right) .
$$

In particular, $L$ generates a holomorphic semi-group $e^{L t}$ in $C^{0}$.

Guckenheimer [1982] showed that there are parameter values for which, at the trivial equilibrium, there is a simple zero eigenvalue of $E_{k}$, pure imaginary eigenvalues $\pm i \gamma$ of $E_{1}$, and all other eigenvalues have negative real parts. This is a consequence of the fact that $\operatorname{tr} E_{n}$ is a monotonically decreasing function of $n^{2}$ and $\operatorname{det} E_{n}$ is strictly convex. If one regards $\left(A^{2}, B\right)$ as being experimental parameters with the diffusion rates $\left(D_{1}, D_{2}\right)$ fixed, then the corresponding conditions are

$$
A^{2}=D_{2} k^{2} \frac{D_{1}+D_{2}-D_{1} k^{2}}{1+D_{1} k^{2}-D_{2} k^{2}}, \quad B=1+A^{2}+D_{1}+D_{2}
$$

subject to the following inequalities on the diffusion rates

$$
\operatorname{det} E_{k \pm 1}>0 \text {. }
$$

There are solutions to (3.4) and (3.5) with $A, B, D_{1}$ and $D_{2}$ all positive. 
Now let $A^{2}$ and $B$ vary in a neighborhood of such a critical point. Then a straightforward computation shows that Langford's condition (2.26) is satisfied. Moreover, the center manifold theorem applies to yield a three-dimensional submanifold of $C_{0}^{2}$ near the origin (depending smoothly on the parameters) which is locally invariant and attractive for the flow induced by (3.3), and which contains all "small" solutions which are bounded for all $t \in \mathbb{R}$, in particular equilibria, periodic orbits and invariant tori near the origin. Hence, the restriction of (3.3) to this submanifold gives a complete description of the dynamics we are interested in. The corresponding vector field $V$ can be transformed to the form (1.1). According to Remark 2.5 it suffices to compute the coefficients $a, b, c, \cdots$ at the critical parameter values, in order to check the hypotheses of $\$ 1$ and 2.

Let $E$ be the three-dimensional eigenspace for $L$ corresponding to the eigenvalues with zero real part and let $P: C_{0}^{2}[0, \pi] \rightarrow E$ be the projection onto $E$. Moreover, let $F$ be the complementary eigenspace, and set $Q=P-\mathrm{id}, P w=x$, and $Q w=y$. Since the center manifold is tangent to $E$ at $w=0$, the Taylor expansions of the vector field $V$ and the restriction of $P(L+N)$ to $E$ agree up to terms of order two. Let us choose a basis for $E$ such that the linear part is given in (complex) Jordan normal form

$$
\left.L\right|_{E}=\left(\begin{array}{ccc}
-i \gamma & 0 & 0 \\
0 & i \gamma & 0 \\
0 & 0 & 0
\end{array}\right), \quad \gamma\left(\operatorname{det} E_{1}\right)^{1 / 2} .
$$

Then the second order terms of $\left.N\right|_{E}$ are given by

$$
\begin{aligned}
N_{2}(x)=\left[\left(x_{1}^{2} \alpha_{11}+x_{2}^{2} \alpha_{22}\right.\right. & \left.+x_{1} x_{2} \alpha_{12}\right) \sin ^{2} \xi+x_{3}^{2} \alpha_{33} \sin ^{2} k \xi \\
& \left.+\left(x_{1} x_{3} \alpha_{13}+x_{2} x_{3} \alpha_{23}\right) \sin \xi \sin k \xi\right]\left(\begin{array}{r}
1 \\
-1
\end{array}\right), \quad x_{j} \in \mathbb{C},
\end{aligned}
$$

where

$$
\begin{aligned}
& \alpha_{11}=\frac{1}{A}\left(\frac{B}{2}\left(1-D_{2}^{2} \gamma^{-2}\right)-A^{2}\left(1-\gamma^{-2} D_{2}\left(1+D_{1}\right)\right)\right)+\frac{i}{A}\left(B D_{2} \gamma^{-1}-A^{2} \gamma^{-1}\left(1+D_{1}+D_{2}\right)\right), \\
& \alpha_{12}=\frac{1}{A}\left(B\left(1+\gamma^{-2} D_{2}^{2}\right)-2 A^{2}\left(1+\gamma^{-2} D_{2}\left(1+D_{1}\right)\right)\right), \\
& \alpha_{33}=\frac{A}{B} k^{4} D_{2}^{2}-A^{3} B \\
& \alpha_{13}=\frac{\sqrt{2}}{A}\left(B D_{2} k^{2}-A^{2}\left(A^{2}+k^{2} D_{2}\right)\right)-\frac{i \sqrt{2}}{A}\left(A^{2} \gamma^{-1}\left(1+D_{1}\right)\left(A^{2}+k^{2} D_{2}\right)-B \gamma^{-1} k^{2} D_{2}^{2}\right), \\
& \alpha_{22}=\bar{\alpha}_{11}, \quad \alpha_{23}=\bar{\alpha}_{13} .
\end{aligned}
$$

Now the coefficients $a, b, c$ in (1.1) can be read off from $\mathrm{PN}_{2}(x)$. We list the result of this computation:

$$
\begin{aligned}
& a=\operatorname{Re} \alpha_{13} \frac{1+i}{\pi} \sqrt{2} \int_{0}^{\pi} \sin ^{2} \xi \sin k \xi d \xi \\
& b=\alpha_{33} \frac{2 \mu}{\pi} \int_{0}^{\pi} \sin ^{3} k \xi d \xi \\
& c=\alpha_{12} \cdot \frac{2 \mu}{\pi} \int_{0}^{\pi} \sin ^{2} \xi \sin k \xi d \xi \\
& \mu=\left(\left(A^{2}+k^{2} D_{2}\right)^{2}-A^{2} B\right)^{-1} k^{2} D_{2} .
\end{aligned}
$$


To compute third order coefficients requires considerably more effort. Here not only $\mathrm{PN}_{3}(x)$ has to be considered but also the second order terms of the center manifold representation and of the Poincaré-Birkhoff transformation are involved. (The techniques used are analogous to those in Marsden and McCracken [1976] and Hassard and Wan [1978].) To be more precise, if the center manifold is given by

$$
y=C(x), \quad x \in E,
$$

and if

$$
x=\tilde{x}+T(\tilde{x})
$$

is the Poincaré-Birkhoff transformation, then we have to compute the (resonant) third order terms of the vector field

$$
\tilde{X}(\tilde{x})=[\mathrm{id}+D T(\tilde{x})]^{-1}[P L \tilde{x}+P L T(\tilde{x})+P N(\tilde{x}+T(\tilde{x})+C(\tilde{x}+T(\tilde{x})))]
$$

Obviously, we have

$$
\begin{aligned}
\tilde{X}_{3}(\tilde{x})= & P N_{3}(\tilde{x})+2 P N_{2}\left(\tilde{x}, T_{2}(\tilde{x})\right)+2 P N_{2}\left(\tilde{x}, C_{2}(\tilde{x})\right) \\
& -D T_{2}(\tilde{x})\left(P L T_{2}(\tilde{x})+P N_{2}(\tilde{x})-D T_{2}(\tilde{x}) P L \tilde{x}\right) .
\end{aligned}
$$

Hence, $T_{2}(\tilde{x})$ and $C_{2}(\tilde{x})$ have to computed.

The contribution of $P N_{3}(\tilde{x})$ to the resonant terms in (1.1) is

$$
\begin{aligned}
& d_{1}=\frac{1}{2 \pi}\left(-3-\gamma^{-2} D_{2}^{2}+2 \gamma^{-1} D_{2}+\left(1+D_{1}\right)\left(-2 \gamma^{-2} D_{2}+\gamma^{-1}+3 \gamma^{-2} D_{2}^{2}\right)\right) \int_{0}^{\pi} \sin ^{4} \xi d \xi, \\
& e_{1}=\frac{1}{\pi}\left(-2 B\left(A^{2}+k^{2} D_{2}\right)\left(1-\gamma^{-1} D_{2}\right)\right. \\
& \left.-\left(A^{2}+k^{2} D_{2}\right)^{2}\left(1-\gamma^{-1}\left(1+D_{1}\right)\right)\right) \int_{0}^{\pi} \sin ^{2} \xi \sin ^{2} k \xi d \xi, \\
& f_{1}=\frac{2 \mu}{\pi}\left(-2\left(A^{2}+k^{2} D_{2}\right)\left(1+\gamma^{-2} D_{2}\left(1+D_{1}\right)\right)-B\left(1+\gamma^{-2} D_{2}^{2}\right)\right) \int_{0}^{\pi} \sin ^{2} \xi \sin ^{2} k \xi d \xi \\
& g_{1}=-\frac{2 \mu}{\pi} B\left(A^{2}+k^{2} D_{2}\right)^{2} \int_{0}^{\pi} \sin ^{4} k \xi d \xi
\end{aligned}
$$

Next we compute the second order terms $C_{2}(\tilde{x})$ in the center manifold representation (3.10). These are determined by the solution of the linear equation

$$
D C_{2}(\tilde{x}) L \tilde{x}=L C_{2}(\tilde{x})+Q N_{2}(\tilde{x}), \quad \tilde{x} \in E .
$$

Setting

$$
C_{2}(\tilde{x})=\sum_{i, j=1}^{3} \beta_{i j} \tilde{x}_{i} \tilde{x}_{j}, \quad \beta_{i j}=\beta_{j i}
$$

and using (3.7), we get the following expressions for the 2-vectors $\beta_{i j}=\left(\beta_{i j}^{1}, \beta_{i j}^{2}\right)$ : 


$$
\begin{aligned}
& \beta_{11}=-\alpha_{11}\left(L_{F}^{-1}+2 i \gamma\right)^{-1} Q\left(\begin{array}{r}
1 \\
-1
\end{array}\right) \sin ^{2} \xi, \\
& \beta_{12}=-\frac{1}{2} \alpha_{12} L_{F}^{-1} Q\left(\begin{array}{r}
1 \\
-1
\end{array}\right) \sin ^{2} \xi, \\
& \beta_{22}=-\alpha_{22}\left(L_{F}^{-1}-2 i \gamma\right)^{-1} Q\left(\begin{array}{r}
1 \\
-1
\end{array}\right) \sin ^{2} \xi, \\
& \beta_{33}=-\alpha_{33} L_{F}^{-1} Q\left(\begin{array}{r}
1 \\
-1
\end{array}\right) \sin ^{2} k \xi, \\
& \beta_{13}=-\frac{1}{2} \alpha_{13}\left(L_{F}+i \gamma\right)^{-1} Q\left(\begin{array}{r}
1 \\
-1
\end{array}\right) \sin \xi \sin k \xi, \\
& \beta_{23}=-\frac{1}{2} \alpha_{23}\left(L_{F}-i \gamma\right)^{-1} Q\left(\begin{array}{r}
1 \\
-1
\end{array}\right) \sin \xi \sin k \xi .
\end{aligned}
$$

Note that

$$
\beta_{12}=\bar{\beta}_{12}, \quad \beta_{33}=\bar{\beta}_{33}, \quad \beta_{11}=\bar{\beta}_{22}, \quad \beta_{13}=\bar{\beta}_{23} .
$$

Inserting (3.16) into $2 \mathrm{PN}_{2}\left(\tilde{x}, C_{2}(\tilde{x})\right)$ yields the following contribution to $d, e, f, g$ in (1.1):

$$
\begin{aligned}
& d_{2}=\operatorname{Re} \frac{2(1+i)}{\pi}\left[\left(\frac{B}{A}\left(1-i \gamma^{-1} D_{2}\right)-A\left(1-i \gamma^{-1}\left(1+D_{1}\right)\right)\right) \int_{0}^{\pi} \beta_{11}^{1} \sin ^{2} \xi d \xi\right. \\
& +\left(\frac{B}{A}\left(1+i \gamma^{-1} D_{2}\right)-A\left(1+i \gamma^{-1}\left(1+D_{1}\right)\right)\right) \int_{0}^{\pi} \beta_{12}^{1} \sin ^{2} \xi d \xi \\
& \left.+A\left(1-i \gamma^{-1} D_{2}\right) \int_{0}^{\pi} \beta_{11}^{2} \sin ^{2} \xi d \xi+A\left(1+i \gamma^{-1} D_{2}\right) \int_{0}^{\pi} \beta_{12}^{2} \sin ^{2} \xi d \xi\right], \\
& e_{2}=\operatorname{Re} \frac{2 \sqrt{2}(1+i)}{\pi}\left[\left(\frac{B}{\sqrt{2} A}\left(1+i \gamma^{-1} D_{2}\right)-\frac{A}{\sqrt{2}}\left(1+i \gamma^{-1}\left(1+D_{1}\right)\right)\right) \int_{0}^{\pi} \beta_{11}^{1} \sin ^{2} \xi d \xi\right. \\
& +\left(\frac{B}{A}\left(A^{2}+k^{2} D_{2}\right)-A B\right) \int_{0}^{\pi} \beta_{13}^{1} \sin k \xi \sin \xi d \xi \\
& \left.+\frac{A}{\sqrt{2}}\left(1+i \gamma^{-1} D_{2}\right) \int_{0}^{\pi} \beta_{33}^{2} \sin ^{2} \xi d \xi+A\left(A^{2}+k^{2} D_{2}\right) \int_{0}^{\pi} \beta_{13}^{2} \sin k \xi \sin \xi d \xi\right], \\
& f_{2}=\operatorname{Re} \frac{4 \sqrt{2} u}{\pi}\left[\left(\frac{B}{A}\left(1-i \gamma^{-1} D_{2}\right)-A\left(1-i \gamma^{-1}\left(1+D_{1}\right)\right)\right) \int_{0}^{\pi} \beta_{13}^{1} \sin \xi \sin k \xi d \xi\right. \\
& +\left(\frac{B}{A}\left(A^{2}+k^{2} D_{2}\right)-A B\right) \frac{1}{\sqrt{2}} \int_{0}^{\pi} \beta_{12}^{1} \sin ^{2} k \xi d \xi \\
& \left.+A\left(1-i \gamma^{-1} D_{2}\right) \int_{0}^{\pi} \beta_{13}^{2} \sin \xi \sin k \xi d \xi+A\left(A^{2}+k^{2} D_{2}\right) \frac{1}{\sqrt{2}} \int_{0}^{\pi} \beta_{12}^{2} \sin ^{2} k \xi d \xi\right], \\
& g_{2}=\frac{4 \mu}{\pi}\left[\left(\frac{B}{A}\left(A^{2}+k^{2} D_{2}\right)-A B\right) \int_{0}^{\pi} \beta_{33}^{1} \sin ^{2} k \xi d \xi+A\left(A^{2}+k^{2} D_{2}\right) \int_{0}^{\pi} \beta_{33}^{2} \sin ^{2} k \xi d \xi\right] .
\end{aligned}
$$



equation

Now we compute $T_{2}(\tilde{x})$. This bilinear form is again determined by a linear

$$
D T_{2}(\tilde{x}) P L \tilde{x}-P L T_{2}(\tilde{x})=\Pi P N_{2}(\tilde{x}), \quad \tilde{x} \in E,
$$

where $\Pi$ denotes projection onto the nonresonant terms. Here the ansatz

$$
T_{2}(\tilde{x})=\sum_{i, j=1}^{3} \gamma_{i j} \tilde{x}_{i} \tilde{x}_{j}, \quad \gamma_{i j}=\gamma_{j i},
$$

leads to the following values for the components of $\gamma_{i j} \in \mathbb{C}^{3}$.

$$
\begin{array}{rlrl}
\gamma_{11}^{1} & =\frac{1-i}{\gamma \pi} \sqrt{2} \alpha_{11} \int_{0}^{\pi} \sin ^{3} \xi d \xi, & \gamma_{22}^{2}=\overline{\gamma_{11}^{1}}, \\
\gamma_{22}^{1}=\frac{i-1}{3 \gamma \pi} \sqrt{2} \alpha_{22} \int_{0}^{\pi} \sin ^{3} \xi d \xi, & \gamma_{11}^{2}=\overline{\gamma_{22}^{1}}, \\
\gamma_{12}^{1}=\frac{i-1}{\sqrt{2} \gamma \pi} \alpha_{12} \int_{0}^{\pi} \sin ^{3} \xi d \xi, & \gamma_{12}^{2}=\overline{\gamma_{12}^{1}}, \\
\gamma_{33}^{1}=\frac{i-1}{\gamma \pi} \sqrt{2} \alpha_{33} \int_{0}^{\pi} \sin ^{2} k \xi \sin \xi d \xi, & \gamma_{33}^{2}=\overline{\gamma_{33}^{1}}, \\
\gamma_{23}^{1}=\frac{i-1}{\gamma \pi 2 \sqrt{2}} \alpha_{23} \int_{0}^{\pi} \sin ^{2} \xi \sin k \xi d \xi, & \gamma_{13}^{2}=\overline{\gamma_{23}^{1}}, \\
\gamma_{13}^{1}=0, & \gamma_{23}^{2}=0, \\
\gamma_{11}^{3}=\frac{\mu i}{\pi \gamma} \alpha_{11} \int_{0}^{\pi} \sin ^{2} \xi \sin k \xi d \xi, & \gamma_{22}^{3}=\overline{\gamma_{11}^{3}}, \\
\gamma_{13}^{3}=\frac{\mu i}{\pi \gamma} \alpha_{13} \int_{0}^{\pi} \sin \xi \sin ^{2} k \xi d \xi, & \gamma_{23}^{3}=\overline{\gamma_{13}^{3}}, \\
\gamma_{12}^{3}=\gamma_{33}^{3}=0 . &
\end{array}
$$

This leads to the following contribution of $2 P N_{2}\left(\tilde{x}, T_{2}(\tilde{x})\right)$ in (1.1):

$$
\begin{gathered}
d_{3}=\operatorname{Re} \frac{(1+i) \sqrt{2}}{\pi}\left[\left(4 \alpha_{11} \gamma_{12}^{1}+2 \alpha_{22} \gamma_{11}^{2}+\alpha_{12}\left(\gamma_{11}^{1}+2 \gamma_{12}^{2}\right)\right) \int_{0}^{\pi} \sin ^{3} \xi d \xi\right. \\
\left.+\alpha_{23} \gamma_{11}^{3} \int_{0}^{\pi} \sin ^{2} \xi \sin k \xi d \xi\right], \\
e_{3}=\operatorname{Re} \frac{(1+i) \sqrt{2}}{\pi}\left[\left(2 \alpha_{11} \gamma_{33}^{1}+\alpha_{12} \gamma_{33}^{2}\right) \int_{0}^{\pi} \sin ^{3} \xi d \xi+4 \alpha_{33} \gamma_{13}^{3} \int_{0}^{\pi} \sin ^{2} k \xi \sin \xi d \xi\right. \\
\left.+2 \alpha_{23} \gamma_{13}^{2} \int_{0}^{\pi} \sin ^{2} \xi \sin k \xi d \xi\right], \\
f_{3}=\frac{2 \mu}{\pi}\left[4\left(\alpha_{11} \gamma_{23}^{1}+\alpha_{22} \gamma_{13}^{2}\right) \int_{0}^{\pi} \sin ^{2} \xi \sin k \xi d \xi\right. \\
\left.+2\left(\alpha_{13}\left(\gamma_{23}^{3}+\gamma_{12}^{1}\right)+\alpha_{23}\left(\gamma_{13}^{3}+\gamma_{12}^{2}\right)\right) \int_{0}^{\pi} \sin ^{2} \sin ^{2} k \xi d \xi\right], \\
g_{3}=\frac{2 \mu}{\pi}\left(\alpha_{13} \gamma_{33}^{1}+\alpha_{23} \gamma_{33}^{2}\right) \int_{0}^{\pi} \sin \xi \sin ^{2} k \xi d \xi .
\end{gathered}
$$


Thus, putting these computations together, we end up with the following formulas for the third order coefficients in the normal form (1.1):

$$
d=\sum_{n=1}^{3} d_{n}, \quad e=\sum_{n=1}^{3} e_{n}, \quad f=\sum_{n=1}^{3} f_{n}, \quad g=\sum_{n=1}^{3} g_{n}
$$

with $d_{n}, e_{n}, f_{n}, g_{n}(n=1,2,3)$ given by (3.14), (3.19) and (3.23), respectively. Here we have used the facts that, according to (3.20), the argument of the bracket in (3.13) is just $[\mathrm{id}-\Pi] P N_{2}(\tilde{x})$ and

$$
\Pi D T_{2}(\tilde{x})[\mathrm{id}-\Pi] P N_{2}(\tilde{x})=0 .
$$

In conclusion, we remark that there are solutions of (3.4) and (3.5), such that hypothesis (H1) of $\S 1$ is fulfilled for $a, b$ and $c$ in (3.11), e.g. for $k=5, D_{1}=0.02$ and $D_{2}=0.09$ (see Guckenheimer [1982]). Moreover, if one considers the coefficients in (3.9) and (3.24) as functions of $D_{1}$ and $D_{2}\left(A^{2}\right.$ and $B$ as in (3.4)), then it is plausible that $\Omega$ in (H2) (see 1.14) does not identically vanish. (The precise verification of this is awkward because the constraints on the variables prohibit an asymptotic analysis. However, the numerical computation of $\Omega$ for any particular parameter values by, for example, appropriate truncation of the Fourier series, seems to be straightforward, but is not undertaken here.) Hence, apart from isolated exceptional values of the diffusion rates $D_{1}$ and $D_{2}$ in this set, both hypotheses $(\mathrm{H} 1)$ and $(\mathrm{H} 2)$ are fulfilled. Thus, for these values, one has, in particular, bifurcation to quasi-periodic orbits lying on invariant tori. If $\Omega>0$, then these quasi-periodic orbits form an asymptotically stable 2-torus.

Acknowledgments. We thank John David Crawford, Marty Golubitsky, John Guckenheimer and Gerard Iooss for useful discussions. We also thank Henk Broer for making his preprints available to us.

\section{REFERENCES}

V. I. ARNOL'D [1965], Small denominators I. Mappings of the circumference onto itself, AMS Translations, Series 2, 46, Providence, RI, pp. 213-284.

N. N. Bogoliubov, J. A. Mitropolski and A. M. SAmoilenko [1976], Method of Accelerated Convergence in Nonlinear Mechanics, Springer-Verlag, New York.

B. L. J. BRAAKSMA AND H. W. BROER [1981], Quasi-periodic flow near a codimension one singularity of a divergence free vector field in dimension four, preprint.

H. BROER [1981a], Formal normal form theorems for vector fields and some consequences for bifurcations in the volume preserving case, Lecture Notes in Mathematics, 898, Springer-Verlag, New York, pp. 54-74. [1981b], Quasi-periodic flow near a codimension one singularity of a divergence free vector field in dimension three, Lecture Notes in Mathematics, 898, Springer-Verlag, New York, pp. 75-89. [1982], Quasi-periodicity in local bifurcation theory, preprint.

A. CHENCINER [1982], Courbes fermées invariantes non normalement hyperboliques au voisinage d'une bifurcation de Hopf degenérée pour difféomorphismes de $\left(\mathbb{R}^{2}, 0\right)$, C. R. Acad. Sci., 294, pp. 269-272.

S. N. Chow and J. K. Hale [1982], Methods of Bifurcation Theory, Springer-Verlag, New York.

S. N. Chow, J. Mallet-PAReT AND J. Yorke [1978], Finding zeros of maps: homotopy methods that are constructive with probability one, Math. Comp., 32, pp. 887-899.

J. D. Crawford [1983], The Hopf bifurcation and plasma instabilities, $\mathrm{Ph}$. D. thesis, Univ. of California, Berkeley.

M. GolubitSKy AND W. F. LANGFORD [1981], Classification and unfoldings of degenerate Hopf bifurcations, J. Differential Equations, 41, pp. 375-415.

M. Golubitsky AND D. SChaEFFER [1979], A theory for imperfect bifurcation via singularity theory, Comm. Pure Appl. Math., 32, pp. 21-98. 
J. GUCKENHEIMER [1980], On quasi-periodic flow with three independent frequencies, preprint.

[1981], On a codimension two bifurcation, Lecture Notes in Mathematics, 898, Springer-Verlag, New York, 99-142.

[1982], Multiple bifurcation problems of codimension two, preprint.

B. D. HASSARD, N. D. KAZARINOFF AND Y. H. WAN [1981], Theory and applications of Hopf bifurcation, London Math. Soc. Lect. Notes \# 41, Camb. Univ. Press, London.

B. HASSARD AND Y. H. WAN [1978], Bifurcation formulae derived from center manifold theory, J. Math. Anal. Appl., 63, pp. 297-312.

M. R. HeRman [1977], Mesure de Lebesgue et nombre de rotation, Lecture Notes in Mathematics, 597, Springer-Verlag, New York, pp. 271-293.

P. Holmes [1980], Unfolding a degenerate nonlinear oscillator: a codimension two bifurcation, Ann. NY Acad. Sci., 357, pp. 473-488.

P. J. Holmes AND J. MARSDEN [1982], Horseshoes in perturbations of Hamiltonian systems with two degrees of freedom, Comm. Math. Phys., 82, pp. 523-544.

L. HörMANDER [1977], Implicit function theorems, Stanford Lectures, Stanford Univ., Stanford, CA.

J. P. KEener [1976], Secondary bifurcation in nonlinear diffusion reaction equations, Studies in Applied Mathematics 55, pp. 187-211.

G. Iooss [1979], Bifurcations of Maps and Applications, North-Holland Mathematics Studies 36. North-Holland, Amsterdam.

G. IOOSS AND W. F. LANGFORD [1980], Conjectures on routes to turbulence via bifurcations, Ann. NY Acad. Sci., 357, pp. 489-505.

W. F. LANGFord [1979], Periodic and steady-state mode interactions lead to tori, SIAM J. Appl. Math., 37, pp. 22-48.

[1981], A review of interactions of Hopf and steady-state bifurcations in Nonlinear Dynamics and Turbulence, G. Iooss and D. Joseph, eds., Pitman, London.

[1982], Chaotic dynamics in the unfoldings of degenerate bifurcations, Proc. International Symposium on Applied Mathematics and Information Sciencs, Kyoto Univ.

W. F. LANGFORD AND G. Iooss [1980], Interactions of Hopf and pitchfork bifurcations, Bifurcation Problems and Their Numerical Solution, Birkhäuser, Berlin, 103-134.

J. E. MARSDen AND M. MCCracken [1976], The Hopf Bifurcation and Its Applications, Applied Mathematical Science, Vol. 19, Springer-Verlag, New York.

J. MOSER [1966], A rapidly convergent iteration method and nonlinear partial differential equations, I and II, Ann. Scuola Norm. Sup. Pisa, 20, pp. 265-315, 499-535.

D. Ruelle AND F. Takens [1971], On the nature of turbulence, Comm. Math. Phys., 20, pp. 167-192.

H. RüsSManN [1979], Konvergente Reihenentwicklungen in der Störungs theorie der Himmelsmechanik Selecta Mathematics V. Springer-Verlag, New York, pp. 92-260.

R. J. SACKER [1965], A new approach to the perturbation theory of invariant surfaces, Comm. Pure Appl. Math., 18 , pp. 717-732.

D. G. SCHAEFFER AND M. A. GolUBITSKy [1981], Bifurcation analysis near a double eigenvalue of a model chemical reaction, Arch. Rat. Mech. Anal., 75, pp. 315-347.

J. SCHEURLE [1982], Bifurcation of quasi-periodic solutions from equilibrium points of reversible dynamical systems, Arch. Rat. Mech. Anal., to appear.

C. L. Siegel AND J. K. Moser [1971], Lectures on Celestial Mechanics, Springer-Verlag, New York.

E. ZEHNDER [1975], Generalized implicit function theorems with applications to some small divisor problems I, Comm. Pure Appl. Math., 28, pp. 91-140. 